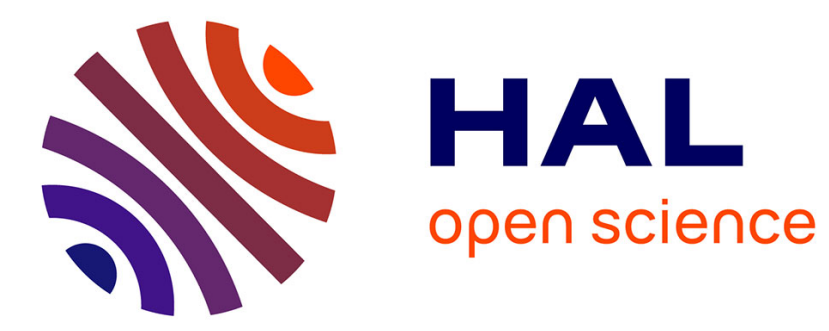

\title{
Color calibration of an RGB digital camera for the microscopic observation of highly specular materials
}

\author{
Juan Martinez-Garcia, Mathieu Hebert, Alain Trémeau
}

\section{To cite this version:}

Juan Martinez-Garcia, Mathieu Hebert, Alain Trémeau. Color calibration of an RGB digital camera for the microscopic observation of highly specular materials. Electronic Imaging 2015, conference: Measuring, Modeling, and Reproducing Material Appearance, Feb 2015, San Francisco, France. 10.1117/12.2075538 . hal-01135969

\section{HAL Id: hal-01135969 \\ https://hal.science/hal-01135969}

Submitted on 27 May 2015

HAL is a multi-disciplinary open access archive for the deposit and dissemination of scientific research documents, whether they are published or not. The documents may come from teaching and research institutions in France or abroad, or from public or private research centers.
L'archive ouverte pluridisciplinaire HAL, est destinée au dépôt et à la diffusion de documents scientifiques de niveau recherche, publiés ou non, émanant des établissements d'enseignement et de recherche français ou étrangers, des laboratoires publics ou privés. 


\title{
Color calibration of an RGB digital camera for the microscopic observation of highly specular materials
}

\author{
Juan Martínez-García*a, Mathieu Hébert ${ }^{\mathrm{a}}$, Alain Trémeau ${ }^{\mathrm{a}}$ \\ a'Université de Lyon, Université Jean Monnet de Saint-Etienne, CNRS UMR 5516 Laboratoire \\ Hubert Curien, F-42000 Saint-Etienne, France
}

\begin{abstract}
Color calibration of imaging devices has been previously studied in a varied number of situations where the materials observed have diffuse or only slightly specular surfaces. Most of the calibration methods available in the literature consist in using standard diffuse color charts in order to determine the mathematical operations necessary to transform the colors measured by the imaging device into the reference colors obtained from the target chart. Unfortunately, there are many problems, such as sensor saturation, that arise when using these methods to calibrate devices intended for the observation of highly specular samples, especially in the $0^{\circ}: 0^{\circ}$ illumination/observation geometry used in microscopic imaging systems. In this paper, we explore several color calibration methods adapted for the observation of highly specular materials, and propose one method in particular in which we use colored filters and a calibrated mirror in order to obtain a set of specular colored samples. By using 72 samples for learning, we tested the different methods on 50 other samples and obtained, with the best one, an average CIE-DeltaE94 color difference of 1.93 units, which is a fairly good performance for color measurements at the microscopic scale.
\end{abstract}

Keywords: Color calibration, color characterization, camera calibration, color chart, color management, specular material, colored transparent filters

\section{INTRODUCTION}

Image acquisition systems provide their users with a practical and inexpensive way to record colored spatial information from a scene or an object. Nevertheless, the colors recorded by such devices might be different from the ones we see, since the spectral responses of the camera sensors are different from the responses of the human visual system ${ }^{1}$. The colors obtained may also vary between different devices due to the fact that the spectral responses of their RGB channels are device dependent; consequently, different recorded colors can be compared with each other only if they were measured with the same imaging system, unless the effective spectral responses of the involved imaging systems are taken into account or if they have been color calibrated. The color calibration of an imaging device consists in building a transformation law between the RGB colors obtained with such device from a set of colored samples (learning samples), and the CIE-XYZ ${ }^{2}$ or CIE-L*a*b*3 values derived from spectral measurements of the same samples, considered as the ground-truth, device-independent colors. One key point for accurate color calibration is that the ground-truth color chart and the objects to be measured must be observed under the same lighting conditions and with the same capture settings for the imaging device. As a consequence, to prevent saturation of the detector or too low light signal, the color chart and the objects need to have comparable reflectances in the selected illumination-observation geometry. Most of the color charts commercially available, such as the GretagMacbeth ColorChecker ${ }^{\circledR}$ or the Calibr8 ColorChart ${ }^{\circledR}$, are Lambertian, or at least very diffusing. They are therefore not adapted to calibrate devices intended to observe specular surfaces, since their reflectance near the specular direction is generally much higher than the reflectance of a diffuse material. An illustration of the distribution of reflected light into different components can be seen in Figure 1. Even when the RGB channels in the calibrated device are not completely saturated due to the high reflected radiance values, the colors recorded will appear clearly brighter than they would actually be perceived by the human visual system. This problem is especially present in the $0^{\circ}: 0^{\circ}$ illumination/observation geometry of a microscope camera since the observation angle is located precisely on the specular reflection angle where the intensity of the light reflected is maximal.

*juan.manuel.martinez.garcia@univ-st-etienne.fr; phone +33 (0) 469663263 


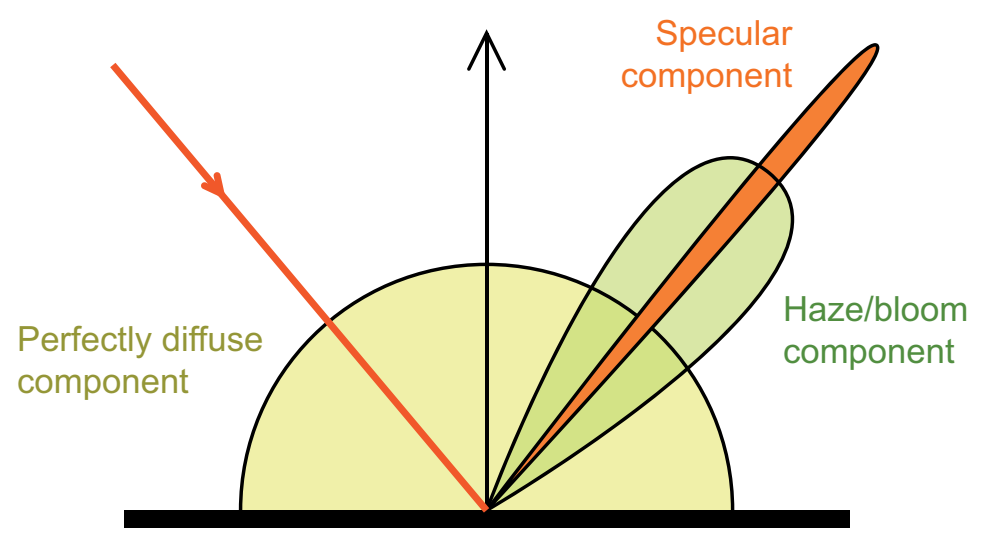

Figure 1. Simplified illustration of the reflective components of materials. Diffuse materials have a large diffuse component and a small specular component, whereas specular materials have a strong specular component and a very small diffuse component.

The first difficulty to overcome when calibrating a device for the observation of specular samples is the unavailability of specular color charts. Besides matte color charts, semi-glossy or glossy color charts such as the GretagMacbeth ColorChecker ${ }^{\circledR}$ SG are not suitable either, because the light reflected by their surface, i.e. the gloss, is mostly achromatic, and it is known that a wide number of well distributed colors are necessary for a good color calibration using polynomial transformations ${ }^{4}$. Moreover, none of the existing color charts are homogenous enough at the microscopic scale to enable the calibration of a microscope camera, which is the aim of our study. In this paper, we thus test four different methods to calibrate an imaging system mounted on a microscope that overcome the aforementioned limitations and show that one of them is an efficient alternative to classical calibration methods, with a comparable accuracy. The metric we will use to express the accuracy of our results is the CIE-DeltaE94 ${ }^{5}$ color difference formula which is one of the most commonly used metrics in the literature and is in better agreement with the human visual system than its widely used predecessor CIE-DeltaE76 ${ }^{3}$.

\section{OPTICAL SETUP}

The imaging device used in our study is an IDS UI-2240-C 8-bit RGB digital camera mounted on an Olympus BX51M microscope, equipped with an Olympus U-TV1x-2 eyepiece with $1 \times$ magnification and a 10× objective lens (MPLN from Olympus). The IDS uEye Cockpit software was used with all its automatic corrections disabled to obtain the RGB images. The samples were illuminated at $0^{\circ}$ (normal to their surface) by a halogen lamp with an optical power of $100 \mathrm{~W}$. This imaging system is used for the microscopic laser inscription of samples that exhibit strong and colored specular reflections, and thus require the type of calibration presented in this paper to correctly capture the colors displayed by the samples. The system has an additional optical entry for the laser beam used for the inscription of the samples and a Melles Griot BTF-VIS-50-5001 M-C 50-50 beam splitter corresponding to this entry.

\section{CALIBRATION SAMPLES}

As the imaging system is intended for the observation of highly specular samples, the different calibration methods were tested with a set of specular samples specifically defined for this study (testing set). In order to obtain a color chart with colored specular reflections, we used 50 colored ROSCO Supergel ${ }^{\circledR}$ filters and a Newport 10D20AL.2 ${ }^{\circledR}$ calibrated mirror. Each of the samples of this color chart is made by placing one of the color filters on top of the calibrated mirror, which allows them to have a strong colored specular reflection. These samples exhibit an adequately regular surface since the microscope is focused on the mirror, which is very homogeneous at microscopic scale.

The color sets used for the learning phase of the calibration vary depending on the method used. There were two different sets of colors used for this purpose: a diffuse color chart and a specular color chart. The diffuse chart was made of 48 pieces of Munsell Matte Color Sheets used in a previous study ${ }^{6}$, composed of 24 colors of similar appearance to the colors of the standard X-Rite ColorChecker ${ }^{\circledR}$ and 24 additional different colors to increase the color space sampling. The specular learning color chart was defined using the same procedure as the specular testing set, i.e., by placing colored 
filters on top of the Newport calibrated mirror, but with a different batch composed of 72 LEE Swatch Book - Numeric Edition ${ }^{\circledR}$ colored filters. The samples ground truth colors in the CIE Chromaticity diagram and in the CIELAB color space are displayed in Figure 2. As it can be seen, for each color set, there is a large number of well distributed samples throughout the color space, which is necessary to obtain a reliable calibration.
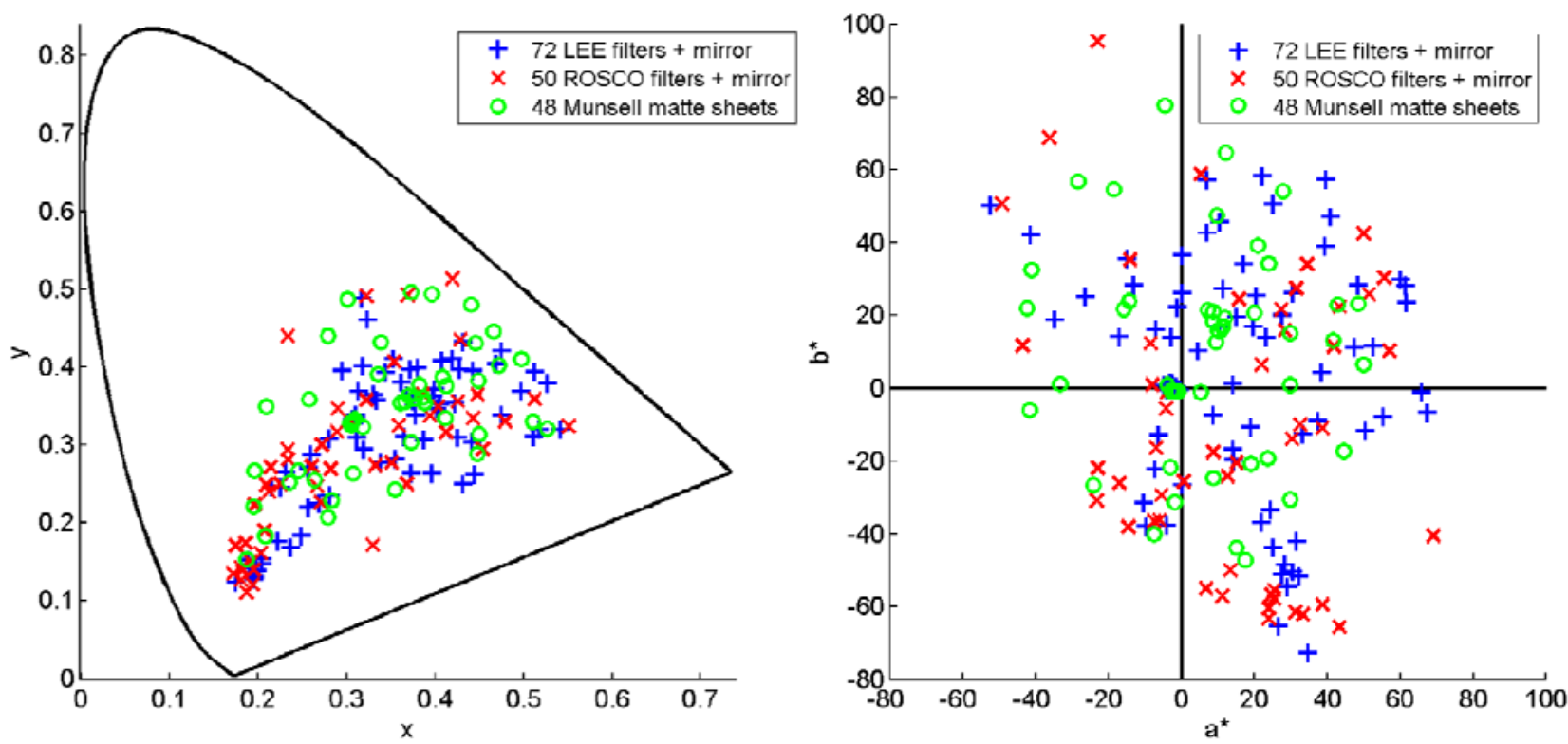

Figure 2. Color sample sets used in this study displayed in the CIE Chromaticity diagram (left) and in the a*-b* plane of the CIELAB color space (right). The CIELAB L* values of the samples range from 22.2 to 96.4 units. For clarity, the illuminant used for these plots was D65.

\section{GROUND TRUTH COLORS OF CALIBRATION SETS}

The color calibration process consists in finding a transformation that converts the colors of the samples measured by the uncalibrated imaging system into the real (ground truth) values of the colors of such samples. In order to obtain the ground truth colors, it is necessary to measure the spectral reflectance of each of the samples in the color sets. This can be done with the use of a spectrophotometer by measuring in the visible part of the electromagnetic spectrum, i.e., the wavelengths from $380 \mathrm{~nm}$ to $780 \mathrm{~nm}$. These spectral reflectance measurements are used to obtain the color values in a device-independent color system to allow comparisons between measurements obtained by different devices. In this study, CIELAB ${ }^{3}$ was chosen as the device-independent color space to represent the colors since it is the most commonly used color space in studies regarding color calibration. Moreover, the CIE-DeltaE94 color difference, which is the metric used to compare the performance of the different methods in this study, is based on the CIELAB color space.

To obtain values in the CIELAB color space, it is necessary to previously calculate the CIE-XYZ values from the spectral measurements, which requires the spectral power distribution of the used illuminant. The microscope system used in this study is equipped with a halogen lamp whose light passes through several optical components. Measuring its exact spectral power distribution was not possible due to the limited space intended for the placement of the samples. Since the light source is an incandescent halogen lamp, and following the recommendation of the $\mathrm{CIE}^{7}$, the standard illuminant A was initially assumed as the illuminant of the microscope. As the illuminant A is an instance of an ideal black body radiator with temperature equal to $2856 \mathrm{~K}$, other different temperatures were also tested in Section 8.2 to evaluate their performance with the different calibration methods proposed.

\section{EXTRACTION OF MEASURED COLORS FROM CAMERA IMAGES}

To obtain the images with the camera, the samples were placed under the microscope and the focus was adjusted by maximizing the sharpness of the image. This adjustment is done each time the position of the sample is changed or a new 
sample is placed for observation. The camera images were stored in files of $1280 \times 1024$ pixels in lossless bitmap image format $^{8}$ (BMP) to avoid color aberrations due to compression. The power of the microscope lamp, the exposure time and the gains of the RGB channels were fixed depending on the calibration method used.

Because the Bayer filters in a color camera are red, green, and blue, and the color images are displayed with red, green, and blue lights on digital displays, the raw values given by the camera are often represented in an RGB space, such as the $\mathrm{sRGB}^{9}$ color space. However, each of these raw values is actually the integral of the spectral power distribution of the light signal multiplied by the spectral response of the detector with its corresponding filter, this latter generally having a large passband in the short, middle or large range of wavelengths. It is therefore comparable, although not similar, to the definition of the CIE-XYZ ${ }^{2}$ color space if we consider the color matching functions in place of the spectral response of the detector with filters. Due to this resemblance and to the fact that the RGB system used by the camera is not specified by the vendor, we consider the raw (uncalibrated) output values of the camera as CIE-XYZ values.

Even though it is hardly noticeably at simple sight, there is a non-uniform irradiance on the images of samples obtained with the microscope. An example of this can be observed in a green sample in Figure 3 (a). As it can be seen, the colors get darker the further away they are from the center of the sample where the distance to the light source is minimal. To extract the color from an image of a color sample, we assume that the most relevant area of the sample is around the center where the light source is illuminating at its maximum power and perform an arithmetic average over all the pixels inside said area. To minimize the effect of sample irregularities or slight geometrical differences, we first perform a pixel-by-pixel average of all the images taken from all the samples in the color set. Afterwards, to further reduce noise we apply a mean filtering over the averaged image, based on a $100 \times 100$ pixel convolution kernel. And finally we convert the pixels to CIELAB colors, select the one with highest luminance $L^{*}$ from them (which we will refer to as $c$ ) and then consider only the pixels whose color difference with $c$ is lower than 5 CIE-DeltaE94 units. An illustration of the area considered for the color extraction for the ROSCO Supergel ${ }^{\circledR}$ color set with the calibrated mirror can be seen in Figure 3 (b). The colors obtained with this procedure are then converted ${ }^{3}$ from the CIE-XYZ color space to the CIELAB color space by using the same illuminant as the one assumed when obtaining the ground truth values.

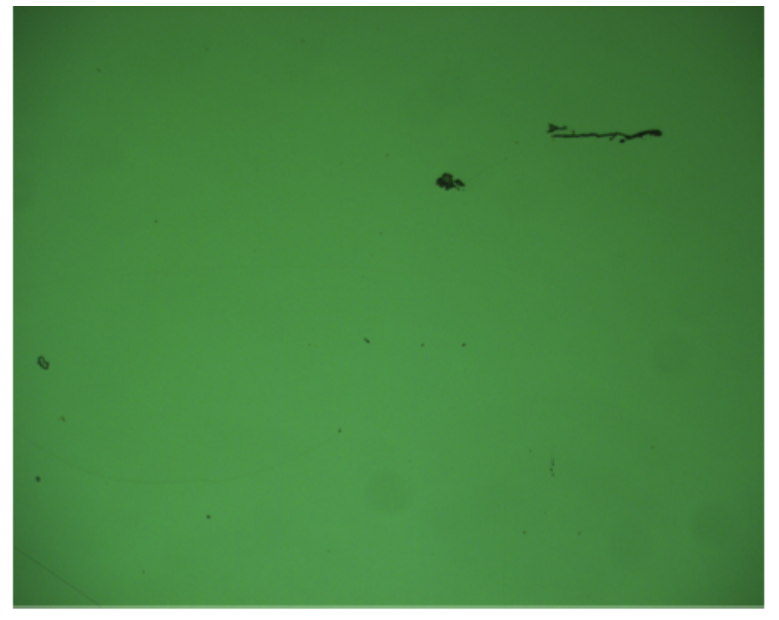

(a)

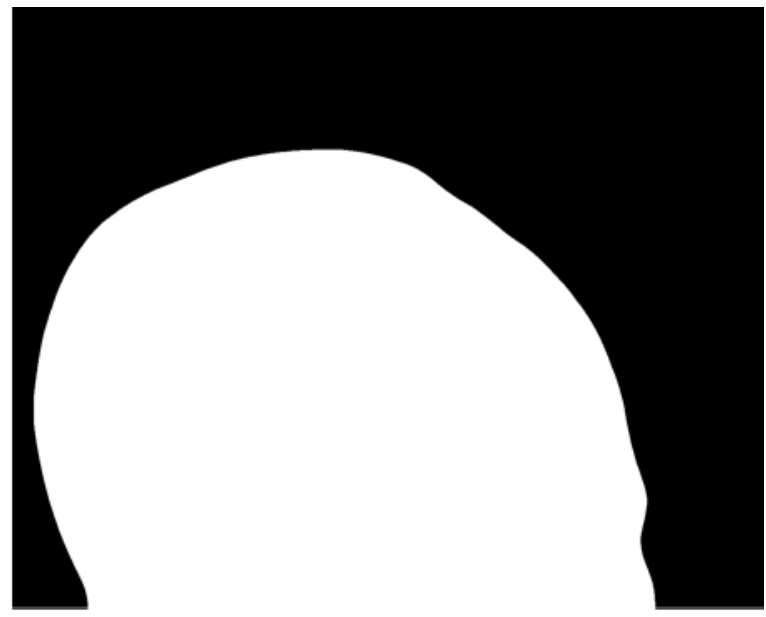

(b)

Figure 3. (a) Non-uniform irradiance on a sample from the learning set consisting of a ROSCO green filter on top of the calibrated mirror. (b) Area considered for the average color extraction of the samples in the learning set consisting of ROSCO color filters on top of the calibrated mirror. The area corresponds to pixels with color difference lower than 5 CIEDeltaE94 units with respect to the pixel with the highest CIELAB L* value.

\section{COLOR TRANSFORMATION}

Several algorithms have been previously studied to calculate the relationship between device dependent and deviceindependent spaces in the color calibration of imaging devices, such as: neural networks ${ }^{10}$, three-dimensional lookup tables $^{11}$, and polynomial transformations ${ }^{4}$. It has been demonstrated that both polynomial transformation and neural 
networks have similar performance even though polynomial transformations are easier to implement and require a smaller number of calibration samples to achieve good results ${ }^{4}$. For that reason, we use a polynomial transformation similar to the one used in a previous study ${ }^{6}$, by also performing, in addition to the 3rd degree polynomial fitting, 2nd and 4th degree fittings in order to study the effects of polynomial overfitting.

\subsection{Polynomial transformation}

The color calibration based on polynomial transformation aims at finding a transformation matrix able to convert the colors measured by the uncalibrated camera into the color of the same samples computed from calibrated spectral reflectance measurements. We thus use a color chart containing several patches. For each patch $i$, we define color vectors in the CIELAB color space. The ground truth CIELAB values obtained from spectral measurements are denoted by the vector $\mathbf{G}_{i}=\left(L_{G, i}, a_{G, i}, b_{G, i}\right)$ and those obtained from the images taken with the uncalibrated camera are denoted by the vector $\mathbf{C}_{i}=\left(L_{i}, a_{i}, b_{i}\right)$. Then, for higher order polynomial regression, an augmented vector $\mathbf{A}_{i}$ is defined from the entries of $\mathbf{C}_{i}$ as shown in Table 1. The matrix $\mathbf{M}$ is the matrix that transforms each vector $\mathbf{A}_{i}$ into a vector $\mathbf{E}_{i}=\left(L_{E, i}, a_{E, i}, b_{E, i}\right)$ whose entries are as close as possible (in the sense of least squares over the calibration set) to the ground truth CIELAB values:

$$
\mathbf{E}_{i}=\mathbf{M} \cdot \mathbf{A}_{i}^{T}
$$

The sizes of vector $\mathbf{A}_{i}$ and of matrix $\mathbf{M}$ depend on the polynomial transformation order. They are given in Table 1 for transformation orders from 1 to 4 .

Table 1. Dimensions of matrix $\mathbf{M}$ and vector $\mathbf{A}$, and elements of vector $\mathbf{A}$ depending on the polynomial order.

\begin{tabular}{cccc}
\hline Order & Size of $\mathbf{M}$ & Size of A & A \\
\hline 1 & $3 \times 4$ & 4 & $(1, L, a, b)$ \\
2 & $3 \times 10$ & 10 & $\left(1, L, a, b, L^{2}, L a, L b, a^{2}, a b, b^{2}\right)$ \\
3 & $3 \times 20$ & 20 & $\left(1, L, a, b, L^{2}, L a, L b, a^{2}, a b, b^{2}, L^{3}, L^{2} a, L^{2} b, L a^{2}, L a b, L b^{2}, a^{3}, a^{2} b, a b^{2}, b^{3}\right)$ \\
4 & $3 \times 35$ & 35 & $\left(\begin{array}{l}1, L, a, b, L^{2}, L a, L b, a^{2}, a b, b^{2}, L^{3}, L^{2} a, L^{2} b, L a^{2}, L a b, L b^{2}, a^{3}, a^{2} b, a b^{2}, b^{3}, \\
L^{4}, L^{3} a, L^{3} b, L^{2} a^{2}, L^{2} a b, L^{2} b^{2}, L a^{3}, L a^{2} b, L a b^{2}, L b^{3}, a^{4}, a^{3} b, a^{2} b^{2}, a b^{3}, b^{4}\end{array}\right)$ \\
\hline
\end{tabular}

The polynomial transformation for each color sample becomes a system of equations with a number of variables equal to the number of entries of $\mathbf{M}$. These entries are calculated by minimizing a function $\sigma$ which corresponds to the sum of the CIE-DeltaE94 color differences between $\mathbf{C}_{i}$ and $\mathbf{E}_{i}$ for all patches $i$ in the learning set, combined with an additional penalty term introduced to favor lower polynomial order solutions and in this way avoid overfitting when possible:

$$
\sigma=\sum_{i=1}^{N} \Delta E_{94}\left(\mathbf{G}_{i}, \mathbf{C}_{i}\right)+\sum_{j=1}^{3} \sum_{k=1}^{N^{\prime}}\left|\mathbf{M}_{j, k} \cdot \operatorname{ord}\left(\mathbf{A}_{i}, k\right) !\right|
$$

where $|p|$ is the absolute value of real number $p, \operatorname{ord}(\mathbf{S}, k)$ denotes the polynomial order of the $k^{\text {th }}$ element of vector $\mathbf{S}$, and $n$ ! is the factorial of integer $n$.

The calculation of the coefficients in the transformation matrix $\mathbf{M}$ in order to minimize $\sigma$ is performed with the iterative function fminsearch of the Matlab ${ }^{\circledR}$ software, which uses the Nelder-Mead Simplex Method. The fminsearch function requires an initial solution in order to improve it towards the local optimum. In the first iteration, we use the least square regression method to minimize de Euclidean distance between $\mathbf{G}_{i}$ and $\mathbf{E}_{i}$ since its computation cost is low and its results are not very far from the optimal values. After each iteration, we keep the best solution found so far and then slightly modify it to use it as a new initial solution for the fminsearch function. The modification consists in introducing some noise to each element by adding a random number between $-10 \%$ and $+10 \%$ of their current value. This mutation range was empirically chosen to allow enough change of the solutions without going unnecessarily far from the optimum. The solutions tend to converge fairly fast and a number of 30 iterations was selected. No significant improvement of the calibration was achieved by using higher number of iterations. 


\subsection{Applying the color calibration}

Once the optimal transformation matrix $\mathbf{M}$ is obtained from the color patches of the learning set, the correction process can be applied to any other measured color, i.e. to each pixel of an image captured by the camera. We assume that the three channels of the camera correspond to uncalibrated CIE-XYZ values. For a given CIE-XYZ color of a pixel $P$ measured by the uncalibrated camera, the following procedure is applied:

1) Convert the pixel values from the CIE-XYZ color space to the CIELAB color space;

2) Compute the augmented vector $\mathbf{A}$ from the obtained CIELAB values depending on the selected polynomial order;

3) Compute the corrected color vector $\mathbf{E}$ by multiplying the transformation matrix $\mathbf{M}$ with the transpose of the vector $\mathbf{A}$ as shown in equation (1);

4) Finally, the corrected CIELAB colors can be converted to and stored in any color space such as the most commonly used digital standard sRGB by applying standard conversions between these color spaces ${ }^{9}$.

\section{METHODS}

As previously mentioned, the purpose of this study is to calibrate a microscopic imaging system intended for the observation of highly specular materials. To accomplish this goal, four different methods were explored: The first two are naïve approaches based on performing a classical color calibration with the use of a diffuse color chart; and the third and fourth methods are more elaborated approaches involving the learning specular color sets defined in this study. All the methods were tested by using the specular color testing set described in Section 3. An overview of the methods described in this study is shown Table 2.

\subsection{Adjustment of exposure time and color channel gains}

In the four methods, a pre-calibration white balance is performed by adjusting the exposure time and the gains of the color channels of the camera in order to decrease the difficulty of the polynomial transformation to find acceptable solutions. The exposure time and color gains in this imaging system are analog settings, and modifying them in the analog domain reduces the quantization errors that could arise when trying to simulate them in the digital domain. The white balance consists in observing the white sample of the current learning color set, followed by adjusting the gains of the color channels until their responses are approximately the same, while also adjusting the time exposure to maximize such responses without saturating any of the channels. The maximization step is done to take advantage of all the dynamic range of the system and minimize the loss of information due to low signal levels and quantization.

\subsection{Ground truth measurement}

To obtain the ground truth values of the color sets in the first, second and third methods, the spectral reflectance of the samples was measured with an X-rite Color $i 7 \AA$ spectrophotometer based on the CIE diffuse d: $8^{\circ}$ sphere geometry. In the fourth method, the spectral reflectance was measured by using the Ocean optics USB-650 spectrophotometer, based on the $0^{\circ}: 0^{\circ}$ illumination/observation geometry with the optical setup described in section 7.6.

\subsection{First method: Diffuse learning set and exposure time decrease}

The first method consists in the following steps:

1) The power of the lamp of the microscope is set to the maximum.

2) The parameters of the camera are adjusted as explained in Section 7.1 by using the white color patch of the learning color set consisting of 48 Munsell matte color sheets.

3) The color transformation matrix $\mathbf{M}$ is calculated by using as input the same learning color set as in Step 2.

4) To test the calibration, the lamp is adjusted to exactly the same power as the one used for the learning set, and the parameters of the camera are adjusted again following the steps in Section 7.1 but this time by using the white patch of the testing set, which in our case is specular. This procedure results in an exposure time lower than the one used for the learning set, in order to normalize the response of the color channels to the higher intensity due to the strong specular reflection. 
5) The transformation matrix $\mathbf{M}$ obtained from the learning set is applied to the testing set camera images with the procedure explained in Section 6.1. Their average colors are extracted and compared to the ground truth colors of the testing color set.

\subsection{Second method: Diffuse learning set and lamp power decrease}

The only difference between the second method and the first method is the step number 4): To test the calibration in the second method, the parameters of the camera are adjusted to exactly the same values as the ones used with the learning set, and in this case the power of the lamp of the microscope is adjusted in order to maximize the responses of the color channels but paying attention not to saturate any of them. Since the testing set consists of specular samples, this will result in a lower lamp power as the one used for the learning set.

\subsection{Third method: Specular learning set}

The steps 1 and 5 are the same as in the previous methods. The remaining steps are as follows:

2) The parameters of the camera are adjusted as explained in Section 7.1 by using the white color patch of the color set consisting of 72 LEE color filters on top of the calibrated mirror.

3) The camera is calibrated by using the same color set as in Step 2.

4) As in this case both the learning and testing sets of colors are specular, to test the calibration, the system is adjusted to exactly the same parameters of the camera and power of the lamp used with the learning set.

\subsection{Fourth method: Optical bench with $0^{\circ}: 0^{\circ}$ geometry}

Lastly, the fourth method is exactly the same as the third method except by the ground truth values of the color sets involved, which were calculated in a different manner. The spectral reflectance measurements were done by using an optical set-up that reproduced the $0^{\circ}: 0^{\circ}$ illumination-observation geometry of the microscope, in which the samples are illuminated and observed at the normal of their surface. This optical measurement configuration is illustrated in Figure 4. The setup consisted of a HL-2000-HP 20W halogen light source from Edmund Optics with an optical fiber whose output was placed in front of a lens to create a collimated beam of light and thus maintain a consistent illumination angle. The light passed through a 50-50 beam splitter before illuminating with a normal incidence angle the sample consisting of the color filter placed in contact with the calibrated mirror. The reflected light from the sample passed again through the beam splitter and then through a telecentric lens made of two lenses and one aperture. Finally, the light passed through an additional lens to focus the beam on an optical fiber connected to a USB2000 spectrophotometer from Ocean Optics.

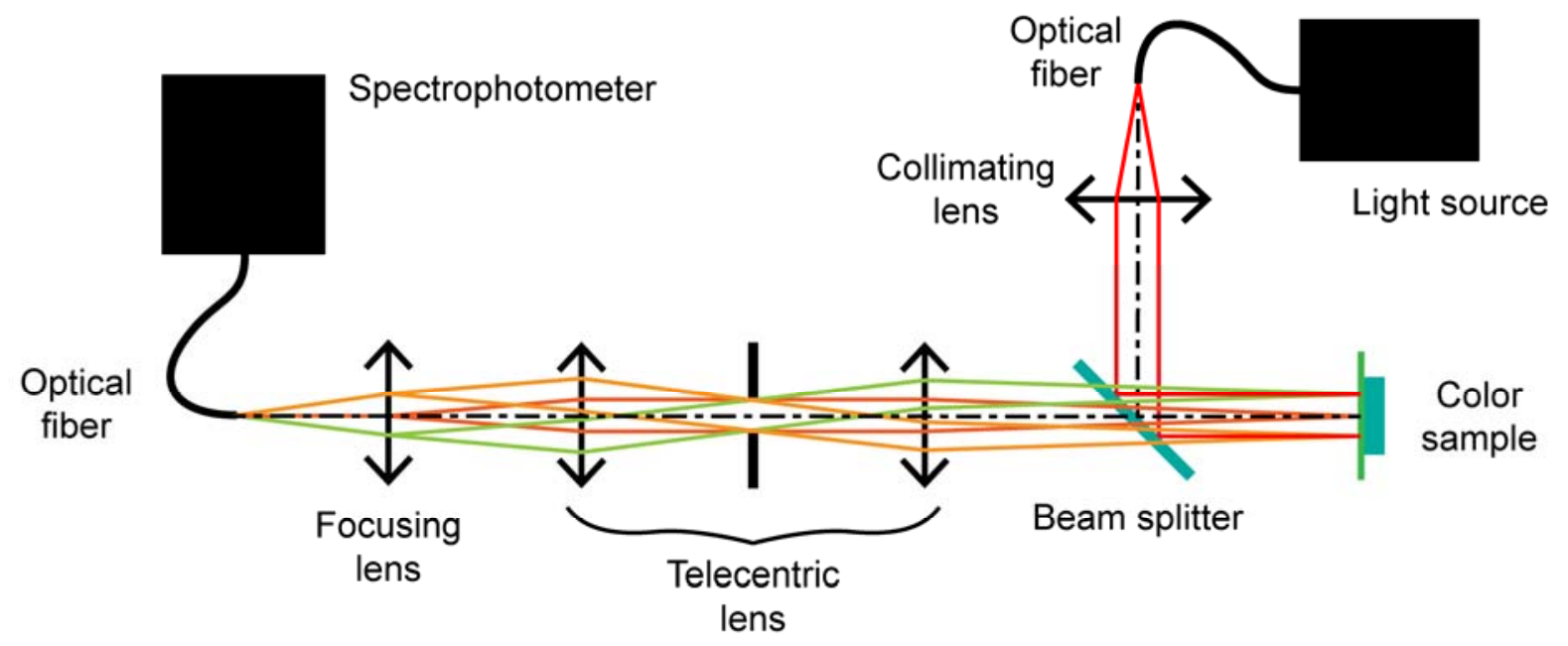

Figure 4. Optical setup based on the $0^{\circ}: 0^{\circ}$ geometry used to obtain the spectral measurements of the samples in order to obtain the ground truth color values with the fourth method. 
Table 2. Overview of the four calibration methods used in this study.

\begin{tabular}{lllll}
\hline & Method 1 & Method 2 & Method 3 & Method 4 \\
\hline Learning set & 48 matte Munsell & 48 matte Munsell & $\begin{array}{l}\text { 72 LEE filters } \\
\text { placed on mirror }\end{array}$ & $\begin{array}{l}\text { 72 LEE filters } \\
\text { placed on mirror }\end{array}$ \\
\hline Testing set & $\begin{array}{l}50 \text { ROSCO filters } \\
\text { placed on mirror }\end{array}$ & $\begin{array}{l}50 \text { ROSCO filters } \\
\text { placed on mirror }\end{array}$ & $\begin{array}{l}50 \text { ROSCO filters } \\
\text { placed on mirror }\end{array}$ & $\begin{array}{l}50 \text { ROSCO filters } \\
\text { placed on mirror }\end{array}$ \\
\hline $\begin{array}{l}\text { Ground truth measurement } \\
\text { device and geometry }\end{array}$ & $\begin{array}{l}\text { X-rite Color } \\
\text { i7® } \mid \text { d:8 } 8^{\circ}\end{array}$ & $\begin{array}{l}\text { X-rite Color } \\
\text { i7® } \mid \mathrm{d}: 8^{\circ}\end{array}$ & $\begin{array}{l}\text { X-rite Color } \\
\text { i7® } \mid \mathrm{d}: 8^{\circ}\end{array}$ & $\begin{array}{l}\text { Ocean optics } \\
\text { USB-650 } 0^{\circ}: 0^{\circ}\end{array}$ \\
\hline Learning time exposure & Initial & Initial & Initial & Initial \\
\hline Testing time exposure & Reduced & Initial & Initial & Initial \\
\hline Learning lamp power & Maximum & Maximum & Maximum & Maximum \\
\hline Testing lamp power & Maximum & Reduced & Maximum & Maximum \\
\hline
\end{tabular}

\section{RESULTS AND DISCUSSION}

The average results of the color calibration obtained with each of the four methods by assuming an A illuminant (i.e. a black body illuminant of $2856 \mathrm{~K}$ temperature) are shown in Table 3. From the four methods used, the third one had the best performance with a significant margin. It is important to note that even though some of the methods give good results regarding their learning sets, they give poor results regarding the testing sets, which can be a consequence of overfitting or errors due to different conditions between the learning and testing conditions. Although the first two methods obtained fairly good results regarding the color set used to obtain the transformation matrix (learning set), the results obtained with the testing color sets were highly unsatisfactory. Therefore, it is evident that the first two naïve methods are not acceptable choices to perform a color calibration of an imaging device intended for the observation of highly specular samples. The first method obtains the transformation matrix from a set of matte samples and afterwards the exposure of the camera is adjusted to the strong reflectivity of specular testing samples. One of the main sources of error of this method can occur if the responses of the sensors are non-linear, in which case the polynomial transformation obtained with the exposure time used with the learning set does not correspond to the polynomial transformation that would be needed for the lower exposure time used with the testing set. On the other hand, the second method obtains the transformation matrix from the same learning set of matte samples and then the power of the lamp of the microscope is decreased in order to adapt the intensity of the reflection from the learning samples to the same exposure time conditions used with the learning set. Some of the main causes of bad performance with this method were the lack of accurate adjustability of the power of the lamp which did not allow a meticulous adjustment with the white sample of the testing set and that then modification of the power of a halogen lamp induces a change in the spectral power distribution of the lamp which in consequence changes the chromaticity of the colors of the samples observed.

Unlike the first two methods, the third and fourth method have unchanged exposure time and lamp power settings throughout the whole calibration process, thus reducing the number of factors affecting the color transformation process, which is reflected in their performance regarding the testing sets. The only difference between the third and fourth method was the device used to measure the ground truth colors of the calibration sets. Despite the fact that the optical setup used in the fourth method resembled more closely the observation/illumination geometry of the microscope's imaging system, the superior performance of the third method could be due to a higher consistency throughout the measurements of all the samples used. The spectrophotometer used in the third method allows the samples to be pressed against the measurement aperture which makes the surface of the color filters parallel to the one of the mirror. In the fourth method the color filters were placed in contact with the mirror but they were not perfectly parallel, allowing the existence of small geometric inconsistencies between measurements in the same color set. In addition, there could have also been small optical misalignments in the setup, which has significant consequences in the case of specular reflectors,. 


\subsection{Influence of the polynomial order}

A higher polynomial order of the calibration allows a better correction of the colors used for the learning phase of the process but it also increases the risk of overfitting, where only the samples fitted are modeled and not the underlying relationship between then, which causes the calibration to make erroneous transformations of colors different from the ones used in the learning phase. This can be clearly seen in the results obtained in this study (Tables 3 and 4), where the higher the polynomial order is, the better the results are regarding the learning color set but the worse the results are regarding the testing set. It is evident that overfitting is present in the fourth order polynomial transformations, hence those results (along with the ones of the first two methods) will be omitted in Section 8.2.

Table 3. Average CIE-DeltaE94 results obtained with each of the four methods by assuming an A illuminant (i.e. a black body illuminant of $2856 \mathrm{~K}$ temperature).

\begin{tabular}{|c|c|c|c|c|c|c|}
\hline \multirow{2}{*}{$\begin{array}{l}\text { Polynomial order } \\
\text { Color set }\end{array}$} & \multicolumn{2}{|c|}{$2^{\text {nd }}$ order } & \multicolumn{2}{|c|}{$3^{\text {rd }}$ order } & \multicolumn{2}{|c|}{$4^{\text {th }}$ order } \\
\hline & Learning $^{a}$ & Testing $^{b}$ & Learning & Testing & Learning & Testing \\
\hline Method 1 & 2.27 & 14.53 & 2.04 & 14.76 & 1.33 & 19.87 \\
\hline Method 2 & 2.28 & 16.73 & 1.60 & 24.92 & 1.48 & 24.98 \\
\hline Method 3 & 2.18 & 2.47 & 1.94 & 2.86 & 1.63 & 3.50 \\
\hline Method 4 & 2.76 & 3.86 & 2.27 & 4.01 & 2.12 & 4.21 \\
\hline
\end{tabular}

\subsection{Influence of the color temperature of the illuminant}

As previously mentioned, the initially assumed color temperature of the illuminant of the imaging system was $2856 \mathrm{~K}$, corresponding to the A illuminant recommended for incandescent light sources. As the actual color temperature of the light source could not be measured, we tested the calibration performance by assuming different color temperatures of an ideal black body radiator and the results for the third and fourth method can be seen in Figure 5. The results suggests that the color temperature of the light source is lower than that of an A illuminant, since the best results were obtained by assuming color temperatures around $1800 \mathrm{~K}$. Taking this into account, we performed the calibration again with the four methods assuming a blackbody illuminant of $1800 \mathrm{~K}$ (Table 4) and achieved an average CIE-DeltaE94 color difference of 1.93 units with the third method using the $2^{\text {nd }}$ polynomial order transformation.

\section{CONCLUSION}

This study was motivated by the necessity of measuring the color of highly specular samples under constrained illumination and observation conditions, imposed by a microscope. A complete comparison between the four methods has been carried out, permitting an interesting discussion on the influence of different parameters on the calibration accuracy of each method, such as the diffusing properties of the color chart, and the polynomial order of the color transformation. Through our experiments, we conclude that the third method with $2^{\text {nd }}$ order degree polynomial fitting is the most accurate: by comparing the colors recorded by the device after transformation and those measured from our set of 50 specular testing samples, we obtained an average CIE-DeltaE94 color difference of 1.93 units, which is a good performance compared with previous studies ${ }^{6,12,13}$, based on diffusing materials where results between 2.2 and 3.0 units of CIE-DeltaE94 color difference were obtained. This method is already being used for color measurements of photochromic dyes created by laser insolation of clear plates ${ }^{14}$. 


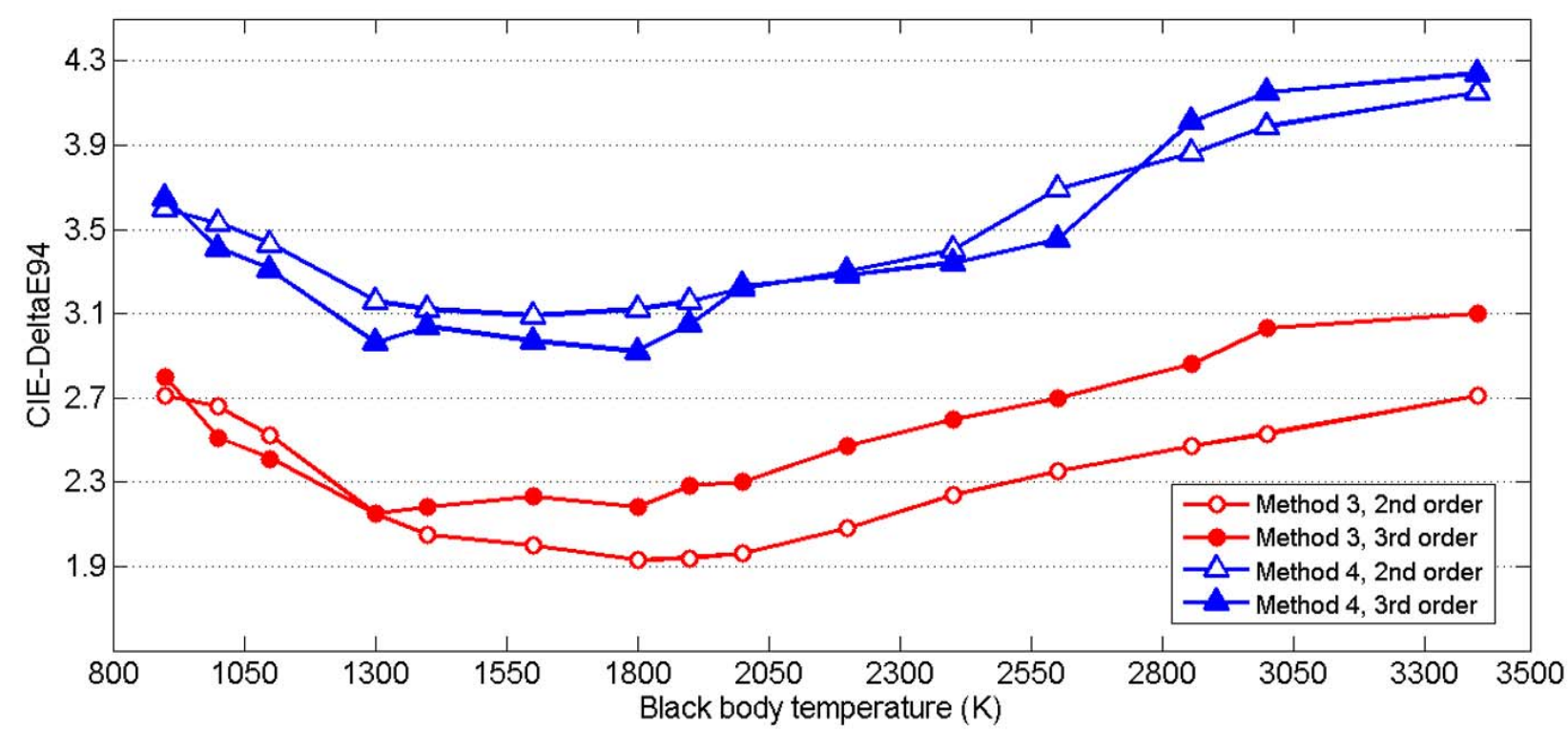

Figure 5. Average calibration results regarding the testing set, by assuming different color temperatures of a black body illuminant with the third and fourth calibration methods.

Table 4. Average CIE-DeltaE94 results obtained with each of the four methods by assuming a black body illuminant of $1800 \mathrm{~K}$ temperature.

\begin{tabular}{lllllll}
\hline Polynomial order & \multicolumn{2}{c}{$2^{\text {nd }}$ order } & \multicolumn{2}{c}{$3^{\text {rd }}$ order } & $4^{\text {th }}$ order \\
\hline Color set & Learning $^{a}$ & Testing $^{b}$ & Learning & Testing & Learning & Testing \\
\hline Method 1 & 2.05 & 15.29 & 1.25 & 20.38 & 1.25 & 20.65 \\
\hline Method 2 & 2.06 & 16.03 & 1.38 & 21.33 & 1.26 & 21.69 \\
\hline Method 3 & 1.87 & $\mathbf{1 . 9 3}$ & 1.64 & $\mathbf{2 . 1 8}$ & 1.46 & $\mathbf{2 . 6 9}$ \\
\hline Method 4 & 2.27 & 3.11 & 1.99 & 3.05 & 1.89 & 3.38 \\
\hline
\end{tabular}

${ }^{a}$ calibration tested with the learning set of samples used to obtain the polynomial transformation matrix.

${ }^{b}$ calibration tested with a different set of samples than the learning set. The best testing results obtained for each polynomial order are highlighted with bold letters.

\section{ACKNOWLEDGMENTS}

This work was supported by the French National Research Agency (ANR) within the program "Investissements d'Avenir" (ANR-11-IDEX-0007), in the framework of project PHOTOFLEX nANR-12-NANO-0006 and the LABEX MANUTECH-SISE (ANR-10-LABX-0075) of Université de Lyon.

\section{REFERENCES}

[1] Sharma, G., [Digital Color Imaging Handbook], CRC Press, (2002).

[2] CIE Central Bureau, "Colorimetry," CIE Pub. 15, Vienna (2004).

[3] Joint ISO/CIE Standard, "CIE Colorimetry - Part 4: 1976 L*a*b* Colour Space," ISO 11664-4 E (2008) / CIE S 014-4 E (2007). 
[4] Cheung, V., Westland, S., Connah, D., and Ripamonti, C., "A comparative study of the characterisation of colour cameras by means of neural networks and polynomial transforms," Color. Technol. 120, 19-25 (2004).

[5] CIE Central Bureau, "Industrial colour-difference evaluation,” CIE Pub. 116, Vienna (1995).

[6] Charrière, R., Hébert, M., Trémeau, A., and Destouches, N., "Color calibration of an RGB camera mounted in front of a microscope with strong color distortion," Appl. Opt. 52, 5262-5271 (2013).

[7] Joint ISO/CIE Standard, "CIE Standard Illuminants for Colorimetry," ISO 10526 (1999) / CIE S 005 E (1998).

[8] Murray, J., [Encyclopedia of Graphics File Formats, 2nd Edition], O'Reilly Media, (1996).

[9] IEC, "Multimedia systems and equipment - Colour measurement and management - Part 2-1: Colour management - Default RGB colour space - sRGB," IEC 61966-2-1 (1999-10) ISBN: 2-8318-4989-6 - ICS codes: 33.160.60, 37.080 - TC 100 - 51 pp. as amended by Amendment A1:2003.

[10] Artusi, A. and Wilkie, A., "Novel color printer characterization model," J. Electron. Imaging 12(3), 448-458 (2003).

[11] Hung, P.-C. "Colorimetric calibration for scanners and media," Proc. SPIE 1448, 164-174 (1991).

[12] Quintana, J., Garcia, R., and Neumann, L., "A novel method for color correction in epiluminescence microscopy,” Comput. Med. Imaging Graph 35, 646-652 (2011).

[13] Jackman, P., Sun, D.-W., and ElMasry, G., "Robust colour calibration of an imaging system using a colour space transform and advanced regression modelling,” Meat Sci. 91, 402-407 (2012).

[14] Destouches, N., Martínez-García, J., Hébert, M., Crespo-Monteiro, N., Vitrant, G., Liu, Z., Trémeau, A., Vocanson, F., Pigeon, F., Reynaud, S., Lefkir, Y., "Dichroic colored luster of laser-induced silver nanoparticle gratings buried in dense inorganic films," J. Opt. Soc. Am. B 31, C1-C7 (2014). 